\title{
Transient Central Diabetes Insipidus in Pregnancy with a Peculiar Change in Signal Intensity on T1-Weighted Magnetic Resonance Images
}

\author{
Tsunehiko Yamamoto, Tomofusa IshII, Katsunobu YoshiokA, Keiko YAmagami, Tetsuya YamaKiTA, \\ Masashi MiYamoto, Masayuki Hosoi, Toshihiko SATo, Shiro TANAKA and Satoru FuJII
}

\begin{abstract}
A 38-year-old woman was admitted with severe thirst and polyuria at 31 weeks' gestation. The plasma concentration of vasopressin (AVP) was very low $(0.73 \mathrm{pg} / \mathrm{ml})$ under conditions of high plasma osmolality $(316 \mathrm{mOsm} /$ kg). T1-weighted magnetic resonance (MR) images revealed enlargement of the pituitary posterior lobe with absence of the hyperintense signal. After delivery, restoration of the hyperintense signal was demonstrated. This depletion-repletion process, which reflects the decrease and increase in amount of neurosecretory granules, is recognized in the case of transient central diabetes insipidus during pregnancy. We consider that an increase in cystine-aminopeptidase (CAP) activity is implicated in the pathogenesis.
\end{abstract}

(Internal Medicine 42: 513-516, 2003)

Key words: diabetes insipidus, pregnancy, posterior pituitary lobe, hyperintense signal, depletion-repletion process

\section{Introduction}

Transient diabetes insipidus, presenting with polyuria and polydipsia during pregnancy, is a very rare clinical condition $(1-4)$. The symptoms usually begin in the third trimester and resolve spontaneously after delivery. This disorder involves both central and nephrogenic diabetes insipidus, with possible mechanisms including the degradation of circulating vasopressin (AVP) by the enzyme vasopressinase, namely cystine-aminopeptidase (CAP), and AVP resistance due to increased production of prostaglandin E2 (5). Fujisawa et al
(6) first reported that the hyperintense signal of the posterior lobe on T1-weighted magnetic resonance (MR) images was absent in patients with typical central diabetes insipidus, which reflects an abnormal reduction in content of the neurosecretory granule containing the vasopressin-neurophysin complex. We encountered a rare case of transient central diabetes insipidus in pregnancy with a peculiar change of signal intensity on T1-weighted MR images.

For editorial comment, see p 459.

\section{Case Report}

In September 1999, a 38-year-old woman was admitted to our department at 31 weeks' gestation with recent development of excessive thirst, polydipsia and polyuria (more than $4 l /$ day). She had obstetric examinations once a month at our hospital and the course of gestation was normal until 30 weeks. She had no past history of pregnancy, pituitary surgery or particular family history. She was alert and oriented. Her skin turgor was reduced and mouth was completely dry. No heart murmur or abnormal respiratory sounds were audible. The abdomen was distended due to her enlarged uterus.

Laboratory data on admission reflects the moderate dehydration as follows: the serum sodium concentration was 160 $\mathrm{mmol} / l$ (normal range in the non-pregnant state: 135-150 $\mathrm{mmol} / l)$, potassium concentration $3.8 \mathrm{mmol} / l(3.5-5.1 \mathrm{mmol} /$ l) and chloride concentration $122 \mathrm{mmol} / l(98-109 \mathrm{mmol} / l)$, respectively. Liver and renal function tests exhibited abnormal pattern moderately; AST (aspartate aminotransferase) $162 \mathrm{IU} / l(8-38 \mathrm{IU} / l)$, ALT (alanine aminotransferase) 172 $\mathrm{IU} / \mathrm{l}(4-44 \mathrm{IU} / \mathrm{l})$, creatinine $1.2 \mathrm{mg} / \mathrm{dl}(0.3-1.1 \mathrm{mg} / \mathrm{dl})$, and uric acid $10.6 \mathrm{mg} / \mathrm{dl}(2.6-6.0 \mathrm{mg} / \mathrm{dl})$. She had no significant abnormality on liver ultrasound. The remainder of her rou-

From the Department of Metabolism and Endocrinology, Osaka City General Hospital, Osaka

Received for publication October 10, 2002; Accepted for publication February 5, 2003

Reprint requests should be addressed to Dr. Tsunehiko Yamamoto, the Department of Metabolism and Endocrinology, Osaka City General Hospital, 2-1322 Miyakojima-hondori, Miyakojima-ku, Osaka 534-0021 
Table 1. Endocrinological Data of Anterior Pituitary on Admission

\begin{tabular}{lrl}
\hline ACTH & $157 \mathrm{pg} / \mathrm{ml}$ & $($ normal*, 0-60) \\
Cortisol & $30.1 \mu \mathrm{gg} / \mathrm{dl}$ & $(5.6-21.3)$ \\
Aldosterone & $117.6 \mathrm{ng} / \mathrm{dl}$ & $(2-13)$ \\
PRA & $39.2 \mathrm{ng} / \mathrm{mlh}$ & $(0.2-2.7)$ \\
TSH & $9.81 \mu \mathrm{U} / \mathrm{ml}$ & $(0.49-4.67)$ \\
Free T3 & $1.9 \mathrm{pg} / \mathrm{ml}$ & $(1.5-3.5)$ \\
Free T4 & $0.8 \mathrm{ng} / \mathrm{dl}$ & $(0.7-1.9)$ \\
GH & $0.58 \mathrm{ng} / \mathrm{ml}$ & $(0.28-8.70)$ \\
PRL & $88.5 \mathrm{ng} / \mathrm{ml}$ & $(1.4-14.6)$ \\
LH & $0.2 \mathrm{mIU} / \mathrm{ml}$ & $(0.2-20.0)$ \\
FSH & $0.2 \mathrm{mIU} / \mathrm{ml}$ & $(0.8-22.9)$ \\
\hline
\end{tabular}

PRA: plasma renin activity. *All normal reference ranges are for the non-pregnant state. Plasma AVP was measured in the sample dissolved in EDTA, to inhibit vasopressinase, using radioimmunoassay after extraction with Sep-Pak $C_{18}$ (14).

tine chemistry workup was normal. Endocrinological data on admission showed that basal levels of anterior pituitary hormones were largely within the normal limits in pregnancy (Table 1). It appears that the patient had mild biochemical primary hypothyroidism, and it is likely that the extremely elevated plasma aldosterone and renin activity level were in response to the volume depletion rather than pregnancy. Maternal blood samples for these tests were obtained from the brachial vein in the supine position early in the morning.

Urinary and plasma osmolality were 89 and $316 \mathrm{mOsm} /$ $\mathrm{kg}$, respectively, with free oral water intake. The plasma AVP level measured by radioimmunoassay was markedly decreased $(0.73 \mathrm{pg} / \mathrm{ml})$ under conditions of high plasma osmolality. A hypertonic saline infusion test totally failed to increase the plasma AVP level (Fig. 1). The CAP activity measured by chromatography (7) had a slightly higher level (326 mU/ml; normal range at 31 weeks' gestation: 80-291 $\mathrm{mU} / \mathrm{ml}$ ). Anti-pituitary, anti-thyroid, anti-nuclear antibodies were also negative. T1-weighted MR images of the pituitary demonstrated enlargement of the posterior lobe (about $5 \times 5$ $\mathrm{mm})$ and absence of hyperintense signal, without thickening of the pituitary stalk, while the anterior lobe showed a high intensity signal (Fig. 2 A-1, A-2). Based on a diagnosis of diabetes insipidus, we treated her with intranasal dDAVP at a daily dose of $10 \mu \mathrm{g}$. Her serum sodium level was gradually decreased to $140 \mathrm{mmol} / \mathrm{l}$ by treatment for 18 days, in accordance with a reduction in urine volume. Liver function tests returned to within the normal range in three weeks, however, treatment with intranasal dDAVP was continued. At 38 weeks' gestation, a healthy female infant with a birth weight of 3,005 $\mathrm{g}$ was born by spontaneous vaginal delivery without complication (Apgar score 9 at 1 minute). Two days after delivery, T1-weighted MR images of the pituitary showed no change in the anterior or posterior lobes (Fig. 2B).

At the seventh postpartum week, pituitary T1-weighted MR images demonstrated that the hyperintense signal of the

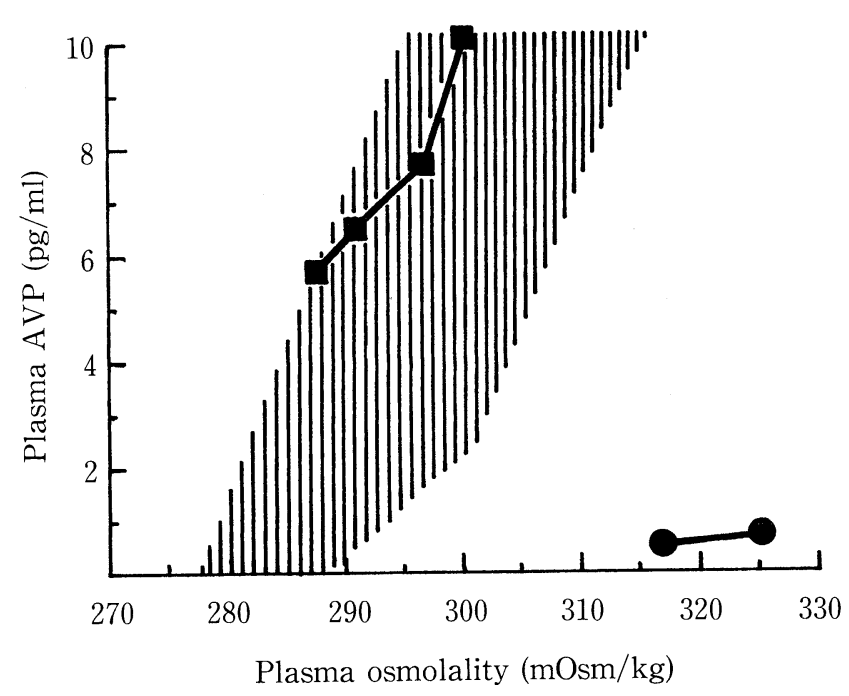

Figure 1. Hypertonic saline infusion test. The patient was given an infusion of a $5 \%$ saline solution at a rate of $0.05 \mathrm{ml} /$ $\mathrm{kg} / \mathrm{min}$ for $120 \mathrm{~min}$. AVP secretion response to $\mathbf{5 \%}$ sodium chloride was low on admission ( () , and recovered completely postpartum ( $\square$ ). The dotted area shows the normal range of the ratio of AVP concentration to plasma osmolality in the nonpregnant state.

posterior lobe had been completely restored with the disappearance of enlargement (Fig. 2C). Since the plasma AVP level had also completely normalized in a hypertonic saline infusion test (Fig. 1), intranasal administration of dDAVP was discontinued. Plasma renin activity and aldosterone level had also completely normalized for the non-pregnant state $(2.2 \mathrm{ng} / \mathrm{m} 1 \mathrm{~h}$ and $10.6 \mathrm{ng} / \mathrm{d} 1$, respectively).

\section{Discussion}

This report describes a woman who had transient diabetes insipidus in late pregnancy. The mechanism responsible for this occurrence is not entirely clear. Soule et al (5) reported that close examination of diabetes insipidus in pregnancy reveals four major subgroups: (1)liver dysfunction promoting reduction of the degradation of vasopressinase, markedly increasing circulating vasopressinase concentrations and consequently the high clearance of vasopressin, (2) permanently following obstetric shock with resultant hypopituitarism, including Sheehan's syndrome, (3) subclinical neurogenic diabetes insipidus with variable degrees of pre-existing vasopressin deficiency such as the existence of pituitary tumor and following previous trans-sphenoidal surgery, (4) nephrogenic diabetes insipidus with concomitant presence of resistance to AVP. We suspected that our patient applied to the first group due to the existence of liver dysfunction and an increase in CAP activity. Whereas acute liver dysfunction in pregnancy is generally caused by either idiopathic events or severe acute fatty liver of pregnancy or pre-eclampsia (8), 


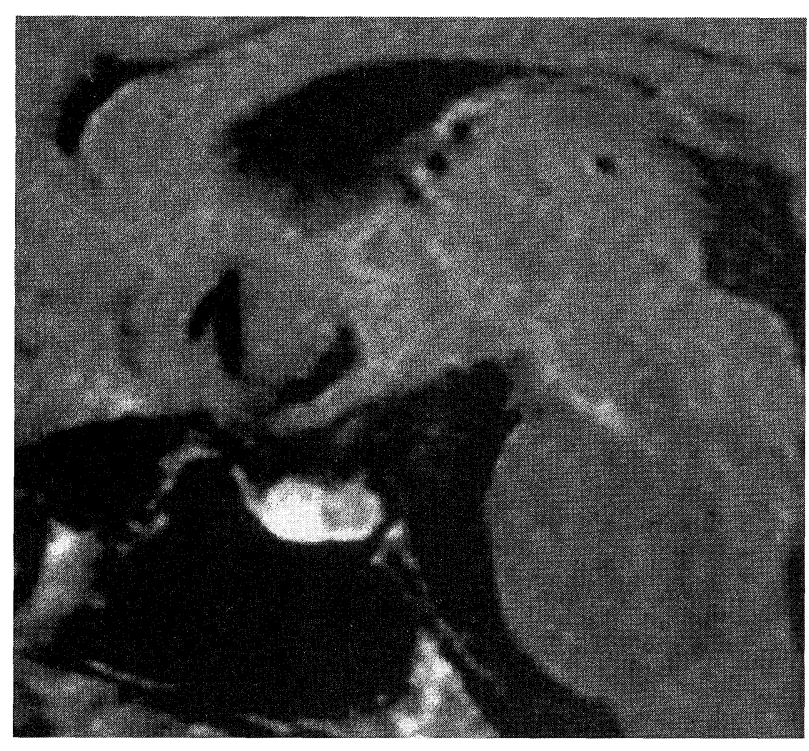

$A-1$

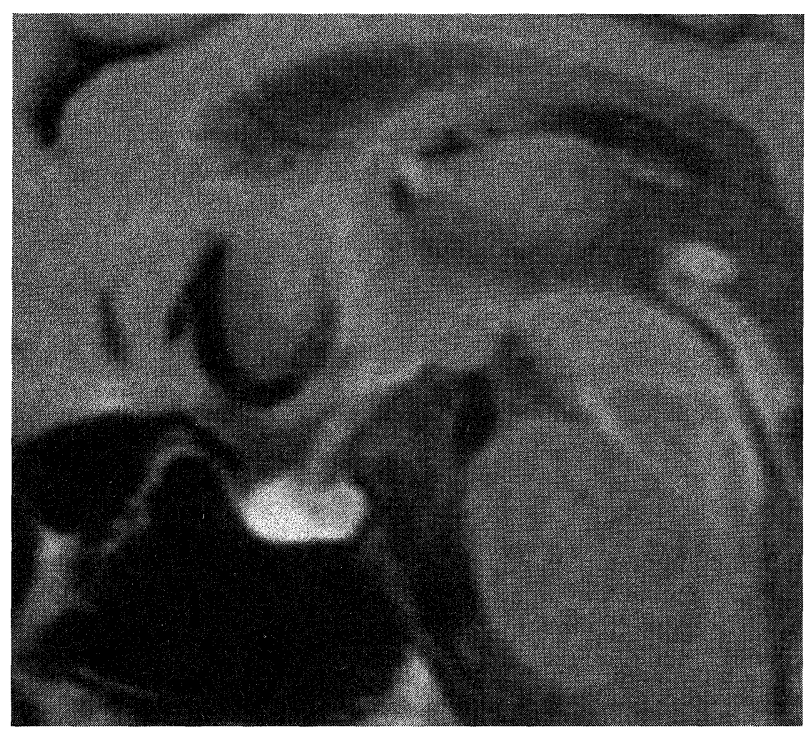

$\mathrm{B}$

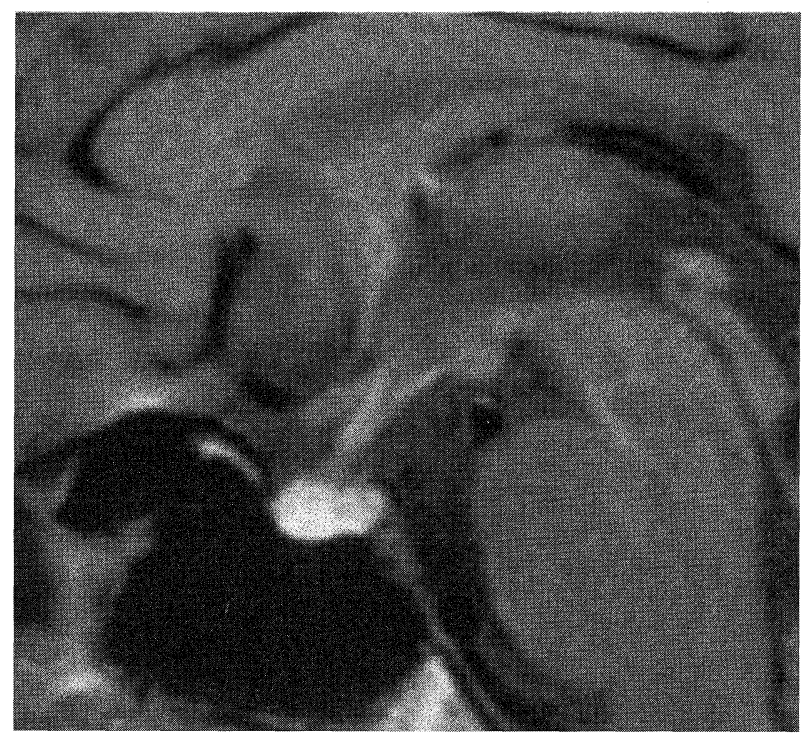

$A-2$

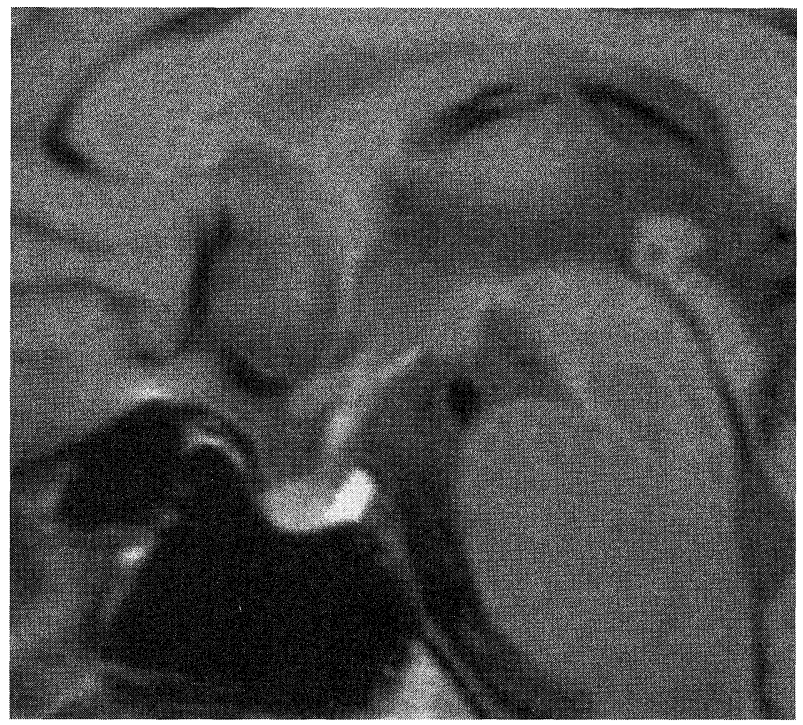

C

Figure 2. T1-weighted MR images of the pituitary (A-1, A-2). Gadolinium was not administered. Pituitary MR images taken at 32 weeks' gestation show enlargement of the posterior lobe $(5 \times 5 \mathrm{~mm})$ and absence of the hyperintense signal, without thickening of the pituitary stalk, while the anterior lobe showed a high intensity signal. (B) Two days after delivery, T1-weighted MR images of the pituitary showed no change in the anterior or posterior lobe. (C) Another pituitary MR image taken in the seventh postpartum week shows that the hyperintense signal of the posterior lobe has been completely restored with remission of enlargement. The anterior lobe is normalized.

the etiology of moderate liver dysfunction could not be definitively determined in our patient. The patient's liver dysfunction did not exert a direct effect on CAP activity, but rather appeared to be secondary to changes in fluid volume balance because she only had moderate liver function on admission. It is possible that an increase in CAP activity due to excessive production in the placenta was involved in the pathogenesis of this case. Although the abnormalities on liver function test gradually returned to normal range in three weeks after starting treatment, preventive administration of dDAVP was continued. It may be correct to state that the patient's needs for dDAVP treatment decreased over the interval. At two days after delivery, T1-weighted MR images still demonstrated enlargement and the absence of the hyperintense signal of the posterior lobe. After restoration of the hyperintense signal and marked normalization of plasma 


\section{YAMAMOTO et al}

AVP response in a hypertonic saline infusion test were confirmed in the seventh postpartum week, intranasal administration of dDAVP was finally suspended.

In healthy individuals, the posterior lobe of the pituitary gland normally demonstrates a characteristically hyperintense signal on T1-weighted MR images. This hyperintense signal is absent when the vasopressin content in the posterior lobe becomes markedly decreased. Based on the present patient's T1-weighted MR examinations, which were performed on admission and in the seventh postpartum week, we were able to evaluate this change in signal intensity, called a depletion-repletion process. It reflects the decrease and increase in amount of neurosecretory granules in the posterior lobe. Recently, in an experimental study using rabbits (9), the hyperintense signal of the posterior lobe essentially disappeared at the end of two weeks of feeding with hypertonic saline solution and reappeared following an additional two weeks of regular water feeding. This study of changes in the posterior pituitary signal in rabbits would be applicable to our patient's situation in that the stimulus for vasopressin production was quite high. In the general depletion process, as demonstrated in these rabbits, there is a greatly enhanced plasma vasopressin level, whereas it is very interesting that the plasma vasopressin level of our patient was markedly low, since increased degradation of vasopressin greatly exceeded the amount secreted in the posterior lobe. The possibility exists that a longer period of this depletion process causes the morphological and pathological changes in the neurohypophyseal axis, enlargement of the posterior lobe and absence of the hyperintense signal. The anterior pituitary lobe of normal late pregnant and postpartum women frequently has a high intensity signal on T1weighted MR images, probably because of a histological increase in prolactin cells (10).

In 1993, Imura et al (11) proposed a new disease entity called "lymphocytic infundibulo-neurohypophysitis (LIN)", which specifically involves neurohypophysis of the pituitary, causing permanent diabetes insipidus. The diagnosis of LIN is usually based on the findings of MR imaging, consisting of thickening of the pituitary stalk and enlargement of the posterior lobe without hyperintense signal on T1-weighted images, which may remit spontaneously in approximately two to seven years $(12,13)$. In the present case, the transient enlargement of the posterior lobe was not accompanied by thickening of the pituitary stalk, which is in conflict with LIN.

We presented here a case report of a woman who developed transient central diabetes insipidus late in pregnancy, which correlated with changes in the posterior pituitary appearance on MR images, and elevated levels of vasopressinase. We postulate that an abnormal increase in vasopressinase activity which subsequently led to low levels of vasopressin and a depletion of vasopressin stores resulted in the absence of the signal, indicating the depletionrepletion process in the posterior pituitary gland on T1weighted MR images.

Acknowledgement: We would like to thank Ichiro Fujisawa, M.D., at the Department of Radiology, Kishiwada City Hospital, for help with the analysis of the T1-weighted MR pituitary images.

\section{References}

1) Barron WM, Cohen LH, Ulland LA, et al. Transient vasopressinresistant diabetes insipidus of pregnancy. N Engl J Med 310: 442-444, 1984.

2) Shah SV, Thakur V. Vasopressinase and diabetes insipidus of pregnancy. Ann Intern Med 108: 435-436, 1988.

3) Hughes JM, Barron WM, Vance ML. Recurrent diabetes insipidus associated with pregnancy: Pathophysiology and therapy. Obstet Gynecol 73: 462-464, 1989.

4) Iwasaki Y, Oiso Y, Kondo K, et al. Aggravation of subclinical diabetes insipidus during pregnancy. N Engl J Med 324: 522-526, 1991.

5) Soule SG, Monson JP, Jacobs HS. Transient diabetes insipidus in pregnancy - a consequence of enhanced placental clearance of arginine vasopressin. Human Reproduction 10: 3322-3324, 1995.

6) Fujisawa I, Nishimura K, Asato R, et al. Posterior lobe of the pituitary in diabetes insipidus: MR findings. J Comput Assist Tomogr 11: 221$225,1987$.

7) Lalu K, Lampelo S, Vahna-Perttula T. Purification of three aminopeptidases from human maternal serum. Int J Biochem 17: 1227-1235, 1985.

8) Krege J, Katz VL, Bowes WA Jr. Transient diabetes insipidus of pregnancy. Obstet Gynecol Surv 44: 789-795, 1989.

9) Fujisawa I, Asato R, Kawata M, et al. Hyperintense signal of the posterior pituitary on T1-weighted MR images: An experimental study. J Comput Assist Tomogr 13: 371-377, 1989.

10) Miki Y, Asato R, Okumura R, et al. Anterior pituitary gland in pregnancy: Hyperintensity at MR. Radiology 187: 229-231, 1993.

11) Imura $H$, Nakao $K$, Shimatsu $A$, et al. Lymphocytic infundibuloneurohypophysitis as a cause of central diabetes insipidus. N Engl J Med 329: 683-689, 1993.

12) Hashimoto $K$, Takao $T$, Makino $S$. Lymphocytic adenohypophysitis and lymphocytic infundibuloneurohypophysitis. Endocr J 44: 1-10, 1997.

13) Maghnie M, Cosi G, Genovese E, et al. Central diabetes insipidus in children and young adults. N Engl J Med 343: 998-1007, 2000.

14) Williams TD, Carter DA, Lightman SL. Sexual dimorphism in the posterior pituitary response to stress in the rat. Endocrinology 116: 738740,1985 